\title{
DRIVER'S BEHAVIOR AND WORKLOAD ASSESSMENT FOR NEW IN-VEHICLE TECHNOLOGIES DESIGN
}

\author{
Annie Pauzié, Julie Manzano \& Nicolas Dapzol \\ INRETS - National Research Institute on Transport and Safety \\ LESCOT- Laboratory Ergonomics \& Cognitive Sciences in Transport \\ Bron/Lyon, France \\ E-mail: pauzie@inrets.fr
}

\begin{abstract}
Summary: Innovative technology can induce improvement in road safety, as long as its acceptability and its adequacy are checked, taking into account the diversified driver's population needs and functional abilities through a Human Centred Design process. Relevant methodology has to be developed in this purpose. Evaluation of the driver's mental workload is an important parameter, complementary to objective ones such as control of the vehicle and driver's visual strategies. This paper describes experiments conducted in the framework of the European project AIDE aiming at validating the DALI (Driving Activity Load Index), a tool set up to allow evaluation of mental workload while using in-vehicle systems; the main results and conclusion from this approach are presented.
\end{abstract}

\section{METHODOLOGY FOR HUMAN CENTRED DESIGN}

\section{Human Centred Design of innovative technologies}

If the driving task has little evolved since the creation of the car, this situation is changing today under the combined effect of widespread of driver information and communication systems (IVIS) and emergence of advanced driver assistance systems (ADAS). In-vehicle devices have to be intuitive, self-explanatory and non intrusive. In order to reach this goal, the human-centred design approach is relevant at each step of the development: setting up the concept, development of the mock-up and the prototype, implementation of the system, with series of iterations to improve the final result (Pauzié A., 2002).

The Network of Excellence HUMANIST (HUMAN centred design for Information Society Technologies), funded by the European Commission DG InfSo, gathers research activities directly linked to this issue: identification of the driver needs in relation to ITS, evaluation of ITS potential benefits, joint-cognitive models of driver-vehicle-environment for user centred design, impact analysis of ITS on driving behaviour, development of innovative methodologies to evaluate ITS safety and usability, driver education and training for ITS use, use of ITS to train and to educate drivers (www.noehumanist.org).

In order to process a human-centred design, it is necessary to investigate deeply the drivers' behavior in relation to the various stages of the driving task: operational (basic vehiclecontrol processes), tactical (choices of vehicle maneuvers according to rules and road environment) and strategic (decisions at high level such as route to follow) in addition to the drivers functional abilities (visual, auditory and cognitive capacities) according to age and 
experience of driving. Identification of drivers' behavior according to new technological development requires several types of investigations, as there is a wide heterogeneity of the population in terms of functional abilities and requirements. Several researches devoted to identification of drivers'needs have been already conducted for functions such as navigation and guidance, Advanced Adaptative Cruise Control, Intelligent Speed Adaptation, Lane Change Assistance (HUMANIST report A.4, 2006).

\section{Evaluation of driver's behavior and functional abilities}

There are discussions and propositions about tools and methods to be developed in order to investigate the impact of system use on road safety according to users population variability. Classically, the parameters to take into consideration in this framework are related to vehicle (trajectory deviations consequent to the system use), drivers' visual strategies (visual demand due to on-board screen) and overall drivers' workload according to the situation. Vehicle deviation trajectories can be a good parameter in relation to visual strategies. Unfortunately from an experimental point of view, and fortunately for road safety, this parameter reveals very high and very rare workload situation, where the driver is on the way to loose control of his vehicle. Some complementary measurements are necessary in order to identify the increase driver's workload with more accuracy than this type of extreme situation.

\section{Evaluation of driver's mental workload}

One of the possible definitions of the workload is that it is the ratio of the task demands to the average maximal capacity for each individual (Rouse W.B., Edwards S.L. \& Hammer J.M. (1993)). To put it in another way, the assessment of workload is coupled with the task difficulty as experienced by the individual (Gopher D. \& Donchin E. (1986). The individual can adapt his behavior to an increased demand of the task, leading for him to more effort and a higher cost, with the consequence of no perceptible effect on the performance. On the contrary, this individual in the same context can adopt the strategy to have a stable level of effort with a decrease of the resulting performance in managing the task. So, objective performance measures are not sufficient by themselves to evaluate the overall constraint of a given situation, evaluation of the corresponding effort for this task is missing to be able to characterize the overall parameters of the context.

In order to measure the individual's mental workload, several approaches are encountered in the literature: measurements of the physiological parameters, method of dual task and method consisting in formalizing the own driver judgment about the workload he experienced. This approach considered as "subjective" has been developed according to various methods such as the S.W.A.T. - Subjective Workload Assessment Technique, the NASA TLX - Task Load Index- (Hart S.G. and Staveland L.E. 1988)...This type of tool allows evaluation rather than measurement by establishing relative comparison between situations.

\section{Subjective Task Load Index}

The mental workload is multidimensional and, among other things, depends upon the type of task. An efficient tool called the NASA-TLX, NASA-Task Load Index, set up by the NASA 
for the evaluation of pilot's workload, has been used for many decades to evaluate subjective mental workload of operators (Matthews R., Legg S., Charlton S., 2003). A modified version of the NASA TLX has been proposed (Pauzié A. \& Forzy J-F, 1996) in order to adapt it to the driving task. As we want to evaluate the workload during a well-defined task, namely the driving task when using an in-vehicle system, we set up a tool focusing on the specific dimensions to take into account for this task. We called it DALI for Driving Activity Load Index.

The NASA TLX assumes that the workload is influenced by mental demand, physical demand, temporal demand, performance, frustration level and effort. After assessing the magnitude of each of the six factors on a scale, the individual performs pair wise comparisons between these six factors, in order to determine the higher source of workload factor for each pair. A composite note quantifying the level of workload is set up by using both factor rating and relative weights computed from the comparison phase.

The basic principle of the DALI is the same than the NASA-TLX, with a scale rating procedure for six pre-defined factors, followed by a weighing procedure in order to combine the six individual scales into a global score. The main difference lies in the choice of the main factors composing the workload score.

Considering the NASA-TLX, one of the factors to be rated is called the Physical component and is usually defined in the following terms: "How much physical activity was required? pushing, pulling, turning, controlling, activating,..." It appears that this question would not be very relevant when considering the driving activity where the control of the vehicle is quite automatic for an experienced driver, and where maneuvers are not supposed to be physically demanding in our nowadays modern cars.

Another example is the mental component defined in the TLX as follows " How much Mental and perceptual activity was required? - thinking, deciding, calculating, remembering, looking, searching,...-". This statement covers both perceptive and cognitive aspects of the workload, and we think it would be interesting in the context of the driving task to be able to identify impact of each of these various modalities.

Finally, the evaluation of the Performance factor can be made using objective data. The subjective rating of a good performance by the driver can show discrepancies with the measured one, but this difference might be due to many other factors than the mental workload itself - low or high self-esteem, motivations to fit to the standard performance,...The procedure to set up the DALI was to ask various experts involved in the driving task studies to define which were, in their opinion, the main factors inducing mental workload for people driving a vehicle equipped with an on-board system (car phone, driving aid system, radio,...).

This tool has been used in two specific ergonomic evaluations conducted in real road situations, aiming at investigating a guidance/navigation system and a hand-free car phone usability by a diversified sample of drivers. 


\section{The Driving Activity Load Index for the evaluation of the driver's mental workload}

Workload in relation to navigation functions and phoning. The DALI has been previously used for the evaluation of a Guidance/Navigation System (Pauzie A., 1994), which allowed to show that the system was presenting an incorrect timing for the auditorymessage display, not adapted to the driving pace maneuvers, and inducing high driver's workload in terms of auditory demand. The DALI values resulting of the comparison between a guidance arrow display versus an electronic map confirmed the fact that the first context was inducing less interference with the driving task for the driver than the second context. The DALI has been also applied to the context of the evaluation of driver's mental workload linked to mobile phone use (Pauzié A. \& Pachiaudi G., 1997). The tool showed a statistically significant impact of the phoning task on the global cost of the driving task, in comparison with a reference situation. The detail of the DALI factors showed that the stronger effect of the phoning was interference with the driving task and auditory demand, inducing also stress for the driver.

Validation of the DALI in the AIDE project. The study has been conducted in the framework of the European project AIDE (Adaptive Integrated Driver-vehicle InterfacE) supported by the DG InfoSo.

a. Evaluation of DALI in real road context. A real road experiment has been conducted in order to define advantages and limits of DALI method for the evaluation of driver's mental workload. If the objective of the experiment was to test tools and methods, then a knowledge a priori of the level of workload induced by the situation is the way to proceed. Indeed, definition of the context will allow to evaluate if the tools reflect correctly what is expected in terms of conditions and in which way the results from the subjective evaluation tool correspond to the workload deliberately induced on the driver.

So, the general principle of the conducted experiment was to set up experimental sessions that are varying objectively in terms of requirements for the driver, inducing then various levels of mental workload to deal with these contexts.

The 4 tested experimental sessions ( 2 situations with a high task demand and 2 situations with a low task demand, both with and without system) were presenting the following characteristics:

- to vary according to the level of workload induced on the driver

- $\quad$ to be as realistic as possible in a context of driving task

In addition to the 6 factors used in the previous studies (Effort of attention, Visual demand, Auditory demand, Temporal demand, Interference, Situational stress), a supplementary factor: Tactile demand, has been used. Proprioceptive perception is not very well known nowadays in the context of the driving task, and there are more and more projects about haptic systems for the driver. In this experiment, the objective was to investigate how this stimulation is perceived by the driver in comparison with the auditory and the visual ones. Theoretically, tactile stimulations are not inducing high level of mental workload, and we 
made this hypothesis a priori. The use of this stimulation in this experiment was an opportunity to evaluate the subjective evaluation tools for this specific case.

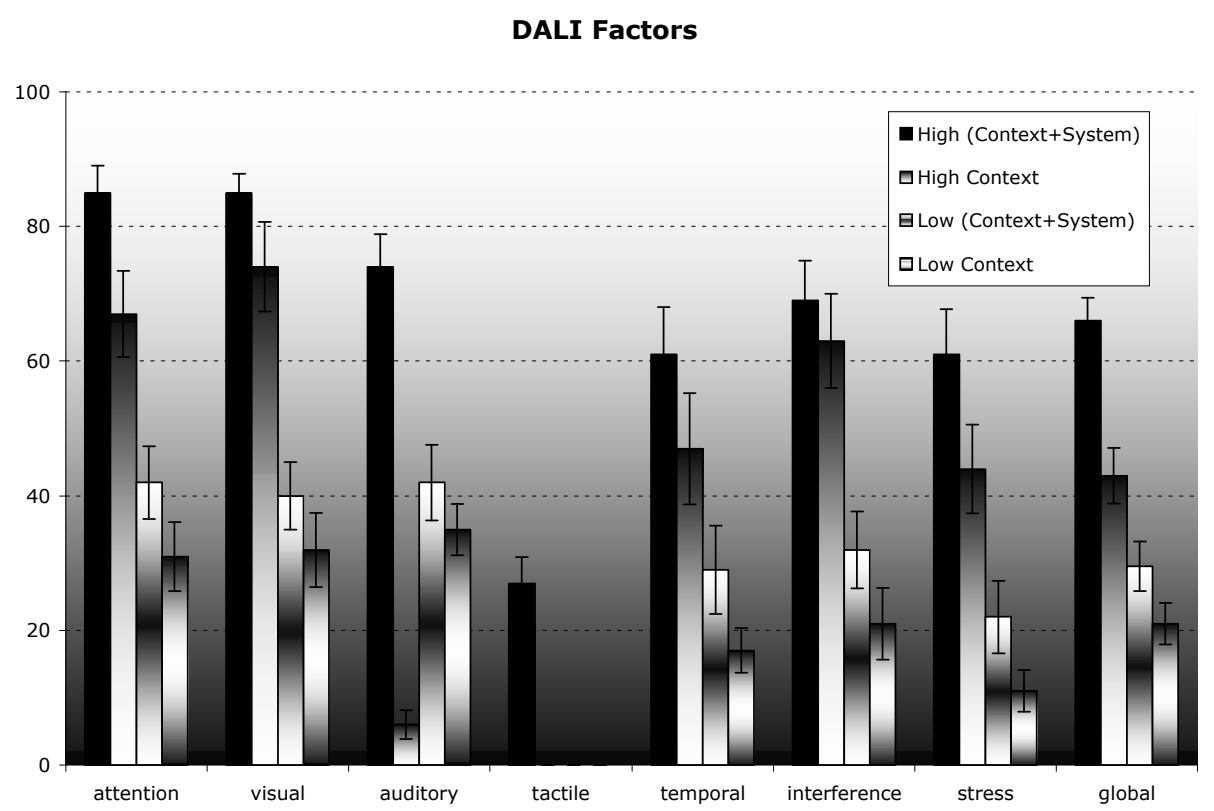

Figure 1. Dali Factors

\section{Global workload}

There is a significant difference between the 4 experimental sessions in terms of subjective assessment of workload by the driver when looking at the DALI results (Wilcoxon, $Z=3,007$, $p=0,003 ; Z=2,224, p=0,026, Z=2,539, p=0,011 ; Z=3,923, p<0,001)$.

These sessions were defined with this goal, so this result is very positive while checking the validity and the sensitivity of this tool.

The chosen sessions were varying according to various characteristics that can participate to this global workload: an analysis of the detail of the results for each factor allows to better identify and understand what are the components of this global score.

\section{Workload linked to cognitive components}

\section{Attention}

There is a significant difference between the High and the Low workload sessions in terms of attentional requirements (Wilcoxon, $Z=2,840, p=0,005 ; Z=3,869, p<0,001$ ). In the High contexts, the attention required to interact with the complex on-board system is higher than the one to find his route according to the memorised information, but the difference is not that significant $(Z=1,991, p=0,047)$. In the Low context, there is no significant difference in 
terms of attention between using a guidance system and following the instructions of a copilot.

\section{Interference}

In terms of interference, there is no significant differences between the High Context With or Without System (between HCS \& HC: Wilcoxon, $Z=0,471, p=0,638$ ), indicating that navigating with a paper map would be rated as interfering with the driving task as using a very complex in-vehicle system or "ergonomic mock-up" displaying several messages and there is no significant difference between the Low Context With or Without System (between LCS \& LC: $Z=1,896, p=0,058$ ), indicating that using a well designed in-vehicle guidance system is equivalent in terms of interference with the driving task to be guided by a human co-pilot. Nevertheless, there is a significant difference when comparing High Context and Low Context, indicating, among other thing, that navigating with a paper map is more interfering for the driving task than using a guidance system(between HC \& LCS:Z=3,037, $\mathrm{p}=0,002$, between HCS \& LCS: $Z=3,662, p<0,001)$.

\section{Stress}

There is a significant difference between most of the different types of driving contexts in terms of stress (Wilcoxon, $Z=2,382, p=0,017 ; Z=2,041, p=0,041, Z=3,880, p<0,001$ ), with a lesser value between the High Context + System and the High Context (Wilcoxon, $Z=$ $1,729, p=0,084)$. The factor stress is reflecting a global evaluation of the task constraint for the driver, and, in a coherent manner, is very low in the situation where the co-pilot is supporting the driver, a bit higher when a guidance system is fulfilling this part, much higher when the driver has to memorise his route and very high when the driver has to manage a secondary task in addition to the driving task.

\section{Workload linked to perceptive components}

\section{Visual Factor}

Considering the visual demand of each session, there is a significant difference between the session with high workload High (Context + System) \& High (Context) and the one with low workload Low (Context + System) \& Low (Context) (Wilcoxon, Z=3,218, p=0,001; $\mathrm{Z}=3,95, \mathrm{p}<0,001)$.

The DALI allows to show there is no significant differences between the 2 sessions "using an on-board system displaying complex stimulations" and "using a paper map to find the route" (Wilcoxon, $Z=1,312, p=0,190 ; Z=1,231, p=0,218$ ). There are also no significant differences between the session "to be guided by a guidance system" and "to be guided by another person". Taking into account the fact that in both situations, the driver relied on the auditory information coming from the system or from the co-pilot, it is relevant to find no significant visual workload in these two contexts. 


\section{Auditory Factor}

Considering the auditory demand of each session, a very low value of workload is displayed in the situation where the driver has to memorise his route with a paper map and to find his way based upon the road directions in comparison with the 3 other situations (significant difference (Wilcoxon, $Z=3,954, p<0,001 ; Z=3,771, p<0,001 ; Z=3,804, p<0,001$ ). Indeed, in this case, even if the general workload of the situation appeared to be high, the DALI results show that the auditory demand is not involved in this workload.

Furthermore, there is no significant difference between the situation "using a guidance system" and following instructions from a co-pilot, showing that the auditory messages coming from the on-board system did not induce a noticeable workload by the driver (Wilcoxon, $Z=1,144, p=0,253$ ).

\section{Tactile Factor}

Implementation of vibrations in the seat of the vehicle was a first approach to define if the driver was able to detect this kind of "unusual" stimulus with accuracy, and if this stimulus was inducing workload. The tactile stimulations were quite well detected and induced a light workload in comparison with situations where this stimulation was non-existence (Wilcoxon, $\mathrm{Z}=3,703, \mathrm{p}<0,001)$. Nevertheless, this workload is far less important than the one induced by auditory and by visual stimulations for the same session.

\section{Workload linked to temporal components}

Like for the global score, for the stress and for the attention, the temporal demand is highly different in relation to the type of session (Wilcoxon, $Z=1,118, p=0,264 ; Z=1,556, p=0,120$, (Wilcoxon, $Z=2,116, p<0,034 ; Z=2,843, p=0,004)$. Indeed, like the other 3 factors, this factor is revealing a global estimation of the cost of the task. As driving task is under time constraint, it is then not surprising to have a workload value in terms of timing closely linked to the level of the task complexity.

b. Summary of main results from the DALI factors. The values of the DALI factors showed the significant difference between the 4 experimental sessions, defined a priori on purpose with an increased level of workload for the driver: this tool allowed in a quick and reliable way to identify the global workload of a given context, and to bring additional precision about the level of load for the vision, the audition, the stress, the attention components for each of these driving contexts.

The values of driver's load (visual, auditory and attentional demands) are not significantly different in the context " using a regular guidance system implemented in the vehicle" and the context of a "co-pilot giving verbal guidance instructions to the driver". These results showed that the implemented system in this case was correctly design in terms of visual and auditory messages (timing, loudness, content) and is not inducing noticeable attentional requirement in terms of management of a secondary task. Nevertheless, the DALI results showed that there is a slightly higher level of stress while using the system in comparison with relying on the 
human co-pilot. These results showed that this tool is sensitive to various aspects of the driving task, and can then support the design process by identifying which part of the task was heavier for the driver. In this specific case, the conclusion would be that the guidance system is correctly design, but that its use requires a phase of familiarisation for the driver to be fully comfortable with it.

The values of driver's load in terms of interference are no significantly different between the High Context With or Without System, indicating that "navigating with a paper map" would be rated as interfering with the driving task as "using a complex ergonomic mock-up" displaying several messages.

The values of driver's load in terms of interference are no significantly different between the Low Context With or Without System, indicating that using a well designed "in-vehicle guidance system" is equivalent in terms of interference with the driving task to be guided by a "human co-pilot."

Nevertheless, there is a significant difference when comparing High Context and Low Context, indicating, among other things, that "navigating with a paper map" is more interfering for the driving task than "using a guidance system".

\section{CONCLUSION}

This tool allowed showing significant differences between the experimental sessions in terms of perceptive, cognitive, stress, temporal demand and interference induced by the driving task.

One of the main advantages is the possibility to identify origins of the driver's workload, allowing then to correct the situation at this identified level (e.g. interference and visual load indicate that an in-vehicle system will have a visual demanding visual display). The possible improvements would be to add factors linked to specific aspect of the driving task useful to evaluate impact of ADAS (e.g. level of stress to keep distance with the vehicle ahead, in the case of a system having an impact on this specificity of the driving task). It is planned to conduct further investigations to improve this method by varying the type of situations. The "DALI tool kit", gathering the detailed method in addition to the automatic computation of the statistics and the display of the graphs, will be soon available on the web site, in order for any researcher to be able to use it in his/her scientific context.

\section{REFERENCES}

Gopher, D., \& Donchin, E. (1986). Workload - an examination of the concept. In K.R. Boff, L. Kaufman \& J.P. Thomas (eds.). Handbook of perception and human performance. Volume II, Cognitive processes and performance. New York: Wiley.

Hart, S.G., \& Staveland, L.E. (1988). Development of the NASA TLX : results of of empirical and theoretical research. In P.A. Hancock and N. Meshkati (eds.) Human Mental Workload. North Holland, Amsterdam, 139-183.

HUMANIST report A.4. (2006). Identification of drivers needs and functional abilities in relation to new ITS systems and services, Deliverable A.4, 138. Available at 
www.noehumanist.org.

Matthews, R., Legg, S., Charlton, S. (2003). Distraction, driving, multiple-task, workload, performance. Accid Anal Prev, 35(4), 451-7.

Pauzie, A. 1994. Human interface of in-vehicle information systems, VNIS' 94, Yokohama, Japan.

Pauzié, A., \& Forzy, J-F. 1996. Ergonomic evaluation of guidance and traffic information in the CARMINAT program, III Annual World Congress on Intelligent Transport Systems, Orlando, CDRom.

Pauzié, A., \& Pachiaudi, G., 1997. Subjective evaluation of the mental workload in the driving context, in Traffic \& Transport Psychology : Theory and Application. T. Rothengatter \& E. Carbonell Vaya (eds.), Pergamon, 173-182.

Pauzié, A. 2002. In-vehicle communication systems: the safety aspect. Injury Prevention Journal, 8, 0-3.

Rouse, W.B., Edwards, S.L., \& Hammer, J.M. (1993). Modelling the dynamics of mental workload and human performance in complex systems. IEEE transactions on systems, man and cybernetics, 23, 1662-1671. 